\section{Herztod-Alarm bei Androgenentzug: Entwarnung}

Hoch kontrovers diskutiert:Treibt die Androgen-Deprivation bei Patienten mit Prostatakarzinom die kardiovaskuläre Mortalität in die Höhe? Nach Alarmmeldungen der letzten Jahre sorgt eine neue Metaanalyse für Beruhigung.

$\mathrm{D}$ ie Androgen-Deprivationstherapie (ADT) mit Agonisten des Gonadotropin-Releasing-Hormons ( $\mathrm{GnRH})$ ist eine wichtige Säule in der Behandlung des fortgeschrittenen Prostatakarzinoms. Weil in - v. a. retrospektiven - Studien eine Zunahme der kardiovaskulären Mortalität auftrat, mahnen einige US-Fachgesellschaften zur Vorsicht, und die Food and Drug Administration hat einen entsprechenden Warnhinweis für GnRH-Agonisten veranlasst.

Eine Metaanalyse von acht prospektiv randomisierten Studien mit 4.141 Patienten bringt jetzt mehr Klarheit. Berücksichtigt wurden nur Studien, die sofortige GnRH-Agonisten-basierte ADT gegen keine oder verzögerte ADT bei Patienten mit nicht metastasiertem, aber prognostisch ungünstigem Prostatakarzinom unter die Lupe nahmen, vollständige Informationen über kardiovaskuläre Todesfälle und eine mediane Beobachtungszeit von mehr als einem Jahr aufwiesen.

Die Zahl kardiovaskulärer Todesfälle zwischen ADT-und Kontroll-Patienten unterschied sich mit einer Inzidenz von 11,0 vs. $11,2 \%$ nicht signifikant. Ähnliches galt für die Studien mit einer über min- destens drei Jahre (11,5 vs. $11,5 \%)$ bzw. nur maximal sechs Monaten laufenden ADT (10,5 vs. 10,3\%). Die Analyse von 4.805 Patienten aus elf Studien mit Daten zur Gesamtmortalität ergab, dass sofortiger Androgenentzug mit signifikant geringerer Prostatakarzinom-spezifischer ( 13,5 vs. $22,1 \%$ ) und Gesamtmortalität ( 37,7 vs. $44,4 \%$ ) einherging.
Fazit: Diese Metaanalyse fand keine Evidenz, dass Androgenentzug das Risiko für kardiovaskuläre Todesfälle bei Prostatakarzinom-Patienten steigert. Die Patienten profitierten jedoch beim Prostatakarzinom-spezifischen und Gesamtüberleben. Unklar bleibt, ob man auch für Patienten mit chronischer Herzinsuffizienz oder Zustand nach Myokardinfarkt Entwarnung geben kann. Ulrike Wepner

Nguyen PL et al. Association of androgen deprivation therapy with cardiovascular death in patients with prostate cancer. JAMA. 2011;306(21):2359-66.
Nicht repräsentative Patientenpopulation

Die kardiovaskuläre Mortalität von Patienten mit nichtmetastasiertem Prostatakarzinom und ungünstiger Prognose ist unter einer Androgendeprivation (ADT) laut der Metaanalyse von Paul L. Ngyen und Kollegen nicht erhöht. Heißt das aber auch, dass die Behandlung keine kardiovaskulären Erkrankungen auslöst oder sie, wenn vorhanden, forciert? Auf diese Fragen gebe die Analyse keine Antworten, bemängeln William K. Kelly und Leonard G. Gomella in ihrem zugehörigen Editorial. Darüber hinaus seien nur randomisierte Studien mit Patienten mit einem vorteilhaften Risiko-Nutzen-Profil berücksichtigt worden - ältere Patienten mit multiplen Komorbiditäten und schlechtem PerformanceStatus kamen nicht vor, obwohl genau sie den größten Teil der Patienten ausmachten. Diese Patienten müssten daher künftig mehr berücksichtigt werden. Bis genauere Daten vorliegen, empfehlen die Kommentatoren, Männer mit Herz-Kreislauf-Erkrankungen während der ADT nach den Vorgaben der American Heart Association zu betreuen.

Kelly WK, Gomella LG. Androgen deprivation therapy and competing risks. JAMA. 2011;306(21):2382-3.

\title{
Androgendeprivation erhöht Thrombosegefahr
}

\section{Prostatakarzinom-Patienten mit - medikamentöser oder chirurgischer - Androgendeprivationstherapie (ADT) erleiden häufiger Thromboembolien (TE). Das Komplikationsrisiko steigt mit der Dauer des Androgenentzugs.}

— ür die retrospektive Kohortenstudie Studie wurden die Versicherungsdaten von 154.611 Patienten mit nichtmetastasierten Prostatakarzinomen analysiert, die ihre Diagnose zwischen 1999 und 2005 im Alter von über 65 Jahren erhalten hatten. 58.466 Männer (38\%) bekamen danach eine ADT, davon $2 \%$ in Form einer bilateralen Orchiektomie.

Binnen median 52 Monaten erlitten 15.950 Patienten mindestens eine TE, das heißt eine tiefe Venenthrombose, eine Lungenembolie oder eine arterielle Em- bolie. Von den Patienten mit ADT waren $15 \%$ betroffen, ohne ADT 7\%. Auch nach dem Abgleich von Patienten- und Tumorcharakteristika ging die ADT mit einem hochsignifikant erhöhten TE-Risiko einher (Hazard Ratio [HR] 1,56; 95\%-Konfidenzintervall 1,50-1,61; $\mathrm{p}<0,0001$ ). Patienten mit Orchiektomie waren noch stärker gefährdet als Patienten mit medikamentöser Androgensuppression (HR 1,97 vs. 1,54). Je länger die ADT dauerte, umso mehr stieg das TE-Risiko: um $40 \%$ bei einer Therapiezeit bis zu einem Jahr, um $66 \%$ bei ein bis drei Jahren und um über $100 \%$ bei mehr als drei Jahren.

Fazit: Wegen der TE-Gefahr müssten individuelle Risikofaktoren wie Adipositas, Rauchen, längere Immobilisierung sowie frühere Schlaganfälle oder TE bei der Entscheidung über eine ADT berücksichtigt werden, so die Autoren, v. a. bei Patienten mit Prostatakarzinom mit niedrigem oder mittlerem Risiko, bei denen der Nutzen der ADT weniger gut belegt ist. Und bei jeder ADT müssten Patienten und Ärzte sich des Risikos bewusst und entsprechend wachsam sein. Beate Schumacher

Ehdaie B et al. Androgen deprivation and thromboembolic events in men with prostate cancer. Cancer 2011; DOI: 10.1002/cncr.26623. 